\title{
Análisis retrospectivo multicéntrico del tratamiento adyuvante con quimioradioterapia en pacientes con cáncer gástrico resecado. Resultados preliminares. Grupo Oncológico del Norte (G.O.N)
}

\author{
E. Vélez de Mendizábal*, E. Estrada**, A. Seral***, M. Zorrilla*, L. Olay**, P. Martínez de Prado****, \\ C. Castañón ${ }^{* * * * *}$, A. Jiménez Lacave ${ }^{* *}$
}

\section{Resumen}

Introducción. En el carcinoma gástrico (CG), los resultados del estudio IT-0116 pusieron de manifiesto el beneficio del tratamiento con quimioterapia más radioterapia adyuvante después de cirugía radical RO, en un grupo de pacientes en los que la linfadenectomía DO (inferior a D1) se había realizado en el $53 \%$ de los casos. Si los resultados de este estudio son trasladables a nuestros pacientes ha sido un punto de controversia.

Material y métodos. Hemos iniciado un análisis retrospectivo multicéntrico de los pacientes con CG que han recibido tratamiento adyuvante según la pauta de IT-0116. Analizamos las características de los pacientes, el tipo de linfadenectomía realizada, el número de ganglios analizados, el tratamiento recibido y la toxicidad.

Resultados. Analizamos 31 pacientes. Se realizó una linfadenectomía D0 en 8(26\%), D1 y entre D1-D2 en 12 (39\%) y D2 en 9 (29\%). Esplenectomía en 2 (6.5\%). La mediana de ganglios analizados fue de 12 (2-38). El 90\% presentaba ganglios positivos. De los 23 pacientes evaluables para la tolerancia al tratamiento, 17 pacientes $(74 \%)$ completaron el tratamiento, 7 (32\%) desarrollaron toxicidad hematológica grado $3-4$ y 5 (20\%) toxicidad digestiva grado 3. La intensidad de dosis del 5 FU fue del $86 \%$ (64-108\%).

Conclusiones. En nuestros hospitales el número de pacientes con linfadenectomías DO es inferior al del estudio IT0116 . Realizaremos análisis de supervivencia tras mayor tiempo de seguimiento.

* Sección de Oncología Médica. Hospital San Millán San Pedro. Logroño

** Servicio de Oncología Médica. Hospital Central de Asturias. Oviedo

*** Servicio de Oncología Radioterápica. Clínica Corachán. Barcelona

**** Servicio de Oncología Médica. Hospital de Basurto. Bilbao

***** Servicio de Oncología Médica. Complejo Hospitalario de León
Background. In gastric cancer, the results of Intergroup 0116 trial showed a benefit for adyuvant chemoradiotherapy after radical surgery. However, in 53\% of this group of patients the lymphadenectomy had been DO (less than D1). Controversy exists as to whether the results of this study can be applied to our patients.

Material and methods. We have conducted a retrospective multicentric analysis including patients with gastric cancer who have received adyuvant treatment with the IT-01 16 regimen after complete resection. We analyze patients characteristics, the extent of lymph-node dissection, the number of cycles administered and toxicity.

Results. Thirty one patients were analyzed. $26 \%$ of the patients underwent a DO dissection. A D1 lymphadenectomy or a lymphadenectomy between D1 and D2 were performed in 12 patients (39\%) and a D2 in 9 patients (29\%). 2 patients were splenectomized. The median number of nodes analyzed were 12 (2-38). 90\% of the patients had positive-nodes. There were 23 patients evaluables for treatment toxicity. 17 patients $(74 \%)$ completed treatment as planned. 7 patients (32\%) developed hematologic toxicity grade $3-4$ and $20 \%$ grade 3 diarrhea. The dose-intensity of fluorouracil was $83 \%(64-100 \%)$.

Conclusions. We have less patients with a DO lymphadenectomy in our hospitals than in the Intergroup trial. We will make a survival analysis after a longer follow-up.

Keys words: Stomach neoplasms. Chemotherapy adjuvant. Radiotherapy. Surgery.

\section{Introducción}

La incidencia del CG ha disminuido en las últimas décadas en occidente'; a pesar de ello, en el mundo ocupa el cuarto lugar en incidencia y el segundo en mortalidad ${ }^{2}$. En España es la segunda causa de muerte por cáncer ${ }^{3}$ y datos europeos recientes muestran que la supervivencia a los 5 años es de un $20 \% 4$.

La cirugía es el tratamiento potencialmente curativo de los pacientes con CG resecable, pero en occidente, la supervivencia de los pacientes tratados con cirugía a los 5 años es del $28 \%{ }^{5}$. Esto pone de manifiesto la necesidad de poder 
contar con un tratamiento adyuvante eficaz. Se han realizado múltiples estudios randomizados, que comparan la cirugía seguida de quimioterapia, frente a cirugía sola. La mayoría de ellos no han podido evidenciar diferencias en la supervivencia. La causa puede estar motivada por una escasa potencia estadística de los estudios o bien por la utilización de regímenes de quimioterapia poco activos. En los últimos años se han realizado 4 metaanálisis ${ }^{6-9}$ encontrándose en los tres últimos un pequeño beneficio de la quimioterapia adyuvante, con un beneficio absoluto en la supervivencia del $4 \%{ }^{10}$

Basados en estudios previos en los que las recidivas locorreginales aparecen en más de un $50 \%$ de los pacientes operados con CG, y trabajando con la hipótesis de que la radioterapia podía contribuir al control de la enfermedad al disminuir las recidivas locales, se diseñó el estudio americano Intergroup IT-0116 en el que se randomizaron 556 pacientes comparando quimiorradioterapia postoperatoria, frente a cirugía sola. El grupo que recibió quimiorradioterapia, al compararlo con el grupo de cirugía sola, presentó una disminución en las recidivas locorregionales y un aumento con significación estadística tanto en la supervivencia libre de recidiva (mediana de 30 frente a 19 meses) como en la supervivencia global (mediana de 36 frente a 27 meses) ${ }^{11}$. Un 54\% de los pacientes presentaron toxicidad hematológica grado $3-4$ y un $33 \%$ toxicidad digestiva grado $3-4$. Se analizó después el tipo de cirugía y sorprendentemente en el $54 \%$ se había realizado una linfadenectomía D0 (disección inferior al nivel N1), en el 36\% D1 y en el 10\% D2.

Desde la publicación de los resultados del estudio IT-0116 en septiembre de 2001, se ha establecido un intenso debate sobre la conveniencia de trasladar estos resultados a la práctica clínica, especialmente en Europa, cuestionándose la utilidad de la radioterapia en los pacientes con linfadenectomías más extensas.

Hemos iniciado un análisis retrospectivo multicéntrico de los pacientes con cáncer gástrico que han recibido tratamiento adyuvante según la pauta de IT-0116, en 5 hospitales del grupo GON.

\section{Material y métodos}

Los criterios de inclusión son pacientes con adenocarcinoma de estómago o de la unión esofagogástrica, RO: resección con intención curativa de todo el tumor con márgenes histológicamente negativos, clasificación patológica AJC 2002 entre IB y IV MO ${ }^{12}$. ECOG<3, función renal y hepática adecuada e ingesta calórica $>1500 \mathrm{kcal} /$ día, que han recibido tratamiento adyuvante según la pauta del IT-0116. Analizamos las características de los pacientes, la cirugía realizada, el número de ganglios analizados, la dosis de quimioterapia y radioterapia recibidas así como la toxicidad.

\section{Tratamiento}

La pauta de tratamiento según el esquema del IT-0116 incluye un primer ciclo con 5 -fluoruracilo: $425 \mathrm{mg} / \mathrm{m}^{2}$, más ácido folínico: $20 \mathrm{mg} / \mathrm{m}^{2}$ I.V.al día, 5 días. A los 28 días después del primer día del ciclo, se inicia radioterapia, consistente en 4500 cGy con un fraccionamiento de $180 c G y$ al día, 5 días por semana, durante 5 semanas. De forma concomi- tante 5 fluoruracilo $400 \mathrm{mg} / \mathrm{m}^{2}$ más ácido fólinico $20 \mathrm{mg} / \mathrm{m}^{2}$ al día, durante los primeros 4 días y los 3 últimos de la radioterapia. Un mes después de finalizada la radioterapia, dos ciclos de 5 días de 5 fluoruracilo $425 \mathrm{mg} / \mathrm{m}^{2}$, más ácido folínico $20 \mathrm{mg} / \mathrm{m}^{2}$ al día, cada 28 días. Reducción de dosis en los pacientes que experimentan toxicidad grado $3 \circ 4^{11}$.

La pauta de radioterapia consiste en 4500 cGy en 25 fracciones, cinco días a la semana, sobre el lecho tumoral, áreas ganglionares regionales y $2 \mathrm{~cm}$ más allá del borde de resección proximal y distal.

\section{Cirugía}

El análisis del tipo de linfadenectomía se ha realizado a partir del informe del protocolo quirúrgico y en base a la definición de la JRSGC (Japanese Research Society for the Estudy of Gastric Cancer) ${ }^{13}$, con las modificaciones correspondientes según la localización del tumor: D1: extirpación completa del nivel I (ganglios cardiales, curvadura menor y mayor, suprapilóricos e infrapilóricos perigástricos de la curvadura mayor y menor). D2: extirpación completa de los niveles I y II (nivel II incluye: ganglios de la arteria gástrica izquierda, arteria hepática común, tronco celíaco y arteria esplénica). D0: inferior a D1.

\section{Resultados}

\section{Características de los pacientes}

Analizamos 31 pacientes incluidos desde octubre de 2000 a noviembre de 2003. Las características de los pacientes y del tumor están expuestas en las Tablas I y II. En el $48 \%$ los tumores eran distales, el 58\% eran T3, ninguno T4. El $90 \%$ presentaban metástasis ganglionares, 10 (32\%) en más de 6 ganglios. Se estudió la relación entre el número de ganglios metastáticos y los examinados ( $N$ ratio) y en el $71 \%$ era superior al $25 \%$.

\section{Cirugía}

El tipo de cirugía y tanto el número de ganglios resecados como el de pacientes con más de 6 ganglios afectos según el tipo de linfadenectomía realizada se expresan en la Tabla III. Hay que destacar que ningún paciente al que se le realizó DO presentaba más de 6 ganglios afectados y que en ese grupo la mediana de ganglios resecados era de 5 (rango:2-16).

TABLA I

\section{Características de los pacientes}

$\mathrm{N}=31$

\section{Edad (años) \\ Mediana \\ Rango}

ECOG entre 0 o $1(\%)$ 


\section{E. Vélez de Mendizábal y cols.}

TABLA II

\begin{tabular}{lc}
\hline \multicolumn{2}{c}{ Características del tumor } \\
\hline \multicolumn{2}{c}{ No31 } \\
\multicolumn{2}{c}{ No de pacientes (\%) } \\
\hline Localización \\
Proximal \\
Cuerpo \\
Distal \\
T \\
T1 & $4(13)$ \\
T2 & $11(35)$ \\
T3 & $15(48)$ \\
T4 & $1(3)$ \\
No. de ganglios positivos & $12(38)$ \\
0 & $18(58)$ \\
$1-3$ & 0 \\
$4-6$ & \\
$>6$ & $3(10)$ \\
N ratio & $11(35)$ \\
$0 \%$ & $7(23)$ \\
$>0-10 \%$ & $10(32)$ \\
$>10-25 \%$ & \\
$>25-100 \%$ & $3(10)$ \\
\hline
\end{tabular}

\section{Tratamiento y toxicidad}

De los 31 pacientes inicialmente incluidos en el estudio, hemos analizado la tolerancia al tratamiento de quimiorradioterapia en 23. Un paciente no inició el tratamiento por fístula postoperatoria, 1 recibió un protocolo diferente, 1 no continuó el tratamiento por problemas sociales, en 2 faltan datos y 3 no han completado todavía el tratamiento. Los 23 pacientes recibieron un total de 102 ciclos de quimioterapia y todos recibieron radioterapia. Completaron el tratamiento con quimioterapia $17(74 \%)$, el resto, 2 recibieron 4 ciclos, uno 3 y otro 1. La mediana de intensidad de dosis de 5-fluo-
TABLA IV

\begin{tabular}{lc}
\hline \multicolumn{2}{c}{ Toxicidad del tratamiento grado 3 y 4} \\
\hline & $\begin{array}{c}\text { Pacientes (\%) } \\
\mathrm{N}=23\end{array}$ \\
\hline Neutropenia 3-4 & $7(32)$ \\
Fiebre y neutropenia & $3(14)$ \\
Digestiva 3-4 & $5(22)$ \\
Vómitos 3-4 & $2(9)$ \\
Mucosistis 3 & $2(9)$ \\
Diarrea 3 & $3(14)$ \\
Tox. Cutánea 3 & $1(4.5)$ \\
\hline
\end{tabular}

ruracilo, con respecto el esquema IT-0116 fue del $86 \%$ (64$108 \%)$.

Las toxicidades grado 3 y 4 están expuestas en la Tabla IV. La mayoría de las neutropenias cursaron sin repercusión clínica. Tres pacientes (17\%) presentaron fiebre y neutropenia. Un paciente desarrolló aplasia medular tardía tras finalizar el tratamiento. Un paciente precisó ingreso por vómitos, mucosistis y diarrea y 2 pacientes perdieron 3 y $7 \mathrm{Kg}$ de peso durante el tratamiento. Uno desarrolló pericarditis y enteritis y otro estenosis de la vía biliar. Durante el tratamiento con radioterapia 5 pacientes $(21 \%)$ presentaron dispepsia grado 1-2. No hubo muertes tóxicas.

\section{Discusión}

En estos momentos, tanto la extensión de la linfadenectomía como el valor del tratamiento adyuvante en el CG operable, son temas de intenso debate a nivel internacional.

Hay diferencias constantes entre Japón y Occidente. En Japón, y a diferencia de lo que ocurre en el resto del mundo, persiste una alta tasa de incidencia, se realizan programas de cribado, los diagnósticos se hacen en estadios más tempranos, las linfadenectomías son más extensas y con menos

TABLA III

Tipo de cirugía, número de ganglios resecados y positivos

\begin{tabular}{|c|c|c|c|}
\hline & $\begin{array}{l}\text { Pacientes } \\
(\%) \mathrm{N}=31\end{array}$ & $\begin{array}{l}\text { Mediana de ganglios } \\
\text { resecado (rango) }\end{array}$ & $\begin{array}{l}\text { No. de pacientes con más } \\
\text { de } 6 \text { ganglios positivos (\%) }\end{array}$ \\
\hline \multicolumn{4}{|c|}{ Tipo de gastrectomía } \\
\hline Subtotal & $21(67)$ & & \\
\hline Total & $10(32)$ & & \\
\hline Esplenectomía & $2(6.5)$ & & \\
\hline \multicolumn{4}{|l|}{ Linfadenectomía } \\
\hline DO & $8(26)$ & $5(2-16)$ & 0 \\
\hline $\mathrm{Dl}$ & $10(32)$ & $13(7-25)$ & $2(20)$ \\
\hline D2 & $9(29)$ & $22(6-38)$ & $3(33)$ \\
\hline Entre D1-D2 & $2(6)$ & $5(5-5)$ & 0 \\
\hline Desconocido & $2(6)$ & $12(12-12)$ & $1(50)$ \\
\hline Total & $31(100)$ & $12(2-38)$ & $10(32)$ \\
\hline
\end{tabular}


mortalidad operatoria, la quimioterapia adyuvante se utiliza de forma más generalizada, y con el mismo estadio los datos de recidiva locorregionales y de supervivencia, son mejores $^{14,15}$. Para explicar este último dato, se han propuesto tres hipótesis: la primera, que estemos ante diferentes enfermedades con diferente curso clínico, la segunda, la migración del estadio producido al aumentar el número de ganglios analizados y la última, que la linfadenectomía más extensa tenga un valor terapéutico añadido. En Japón se utiliza de forma sistemática la linfadenectomía D2. En Europa se han realizado dos ensayos randomizados que comparan la linfadenectomía D2 con D1, uno holandés y otro británico. En ninguno de los dos se detectaron diferencias en la supervivencia a los 5 años ${ }^{16,17}$ y sí una morbilidad y mortalidad más elevada en los pacientes asignados a linfadenectomía D2, en el estudio holandés ( $25 \%$ y $4 \%$ en D1 y $43 \%$ y $10 \%$ en D2). En este estudio, una vez excluidas las muertes postoperatorias, el riesgo de recidiva disminuía del $43 \%$ en D1 al $37 \%$ en D2 ${ }^{18}$. El número de esplenectomías fue mayor en D2, $37 \%$ frente a $11 \%$ en D1. Debido a que la esplenectomía en varios estudios se identifica como el factor más importante asociado a mortalidad postoperatoria, es posible que ésta haya sido la causa por la que ambos estudios europeos no han podido detectar un pequeño beneficio derivado de la linfadenectomía D2 ${ }^{19}$. Series europeas más recientes con linfadenectomías D2 muestran una menor mortalidad ${ }^{20}$.

En nuestra serie se realizó linfadenectomía D0 en el 26\%, D1 o entre D1-2 en el $38 \%$ y D2 en el $29 \%$, situación diferente a la del estudio IT-0116 en la que DO se realizó en el $54 \%$ y D2 en el 10\%. El número de ganglios analizados oscila en un rango muy amplio, pero la mediana de ganglios analizados de 12 la podemos considerar relativamente baja, ya que se necesitan 15 para poder realizar un adecuado estudio de extensión TNM. En dos series occidentales con lifadenectomía D2 la mediana de ganglios analizados es de 24 y $27^{21}, 22$. No disponemos de este dato en el estudio del IT0116 . La tolerancia al tratamiento de nuestra pequeña serie, es posible que sea algo mejor ya que completaron el tratamiento el $74 \%$ de los paciente y presentaron toxicidad grado 3-4 hematológica el $32 \%$ y digestiva el $20 \%$. En la serie del IT-0116 completaron el tratamiento el $60 \%$ de los pacientes y presentaron toxicidad grado 3-4 hematológica y digestiva el $54 \%$ y el $33 \%$, con $1 \%$ de muertes tóxicas.

Es difícil saber si los pacientes a los que se les ha realizado cirugía radical RO en nuestros hospitales se benefician de igual manera del tratamiento con quimiorradioterapia que los del estudio IT-0116 y si el beneficio compensa la toxicidad. El riesgo de recidiva en nuestra serie es alto, el $90 \%$ presentan ganglios positivos, y la razón entre número de ganglios afectados y el número de ganglios resecados ( $\mathrm{N}$ ratio), es mayor del $25 \%$ en el $70 \%$ de los casos. La $\mathrm{N}$ ratio se ha identificado como el factor pronóstico más importante por análisis multivariante, y puede evitar posibles factores de confusión relacionados con el número de ganglios resecados así como la variabilidad individual en el número de ganglios regionales existentes. En una serie italiana con linfadenectomía D2, la supervivencia a los 5 años de los pacientes según la " $\mathrm{N}$ ratio" era del $82 \%, 61 \%, 50 \%$ y $14 \%$, en los pacientes con "N ratio" de 0\%, 1-10\%, 11-25\% y >25\% En estos momentos sabemos que la supervivencia mejora con quimiorradioterapia en pacientes con D0 o Dl de alto riesgo, pero desconocemos si esto ocurre también con D2. La duda es si el beneficio obtenido por la radioterapia se sustituye con una linfadenectomía más extensa, o bien tiene un valor terapéutico añadido incluso después de D2. En el carcinoma de recto, finalmente se ha comprobado que la radioterapia sigue disminuyendo las recidivas locorregionales después de realizar una resección mesorrectal ${ }^{23}$. Un análisis posterior del tratamiento quirúrgico del estudio IT-0116, no detectó interacción del tipo de linfadenectomía sobre el efecto del tratamiento adyuvante, sin embargo, debido a que el número de pacientes con D2 era pequeño, la potencia de la prueba estadística para detectar la interacción era baja. En estos momentos y debido al alto riesgo de recidiva que presentan nuestros pacientes, parece razonable continuar ofreciéndoles la opción de tratamiento adyuvante con quimioradioterapia a pesar del riesgo de toxicidad.

Conocemos por los estudios realizados en la enfermedad avanzada, regímenes de poliquimioterapia más activos ${ }^{24,25}$, que ya se han trasladado a los estudios de la enfermedad localizada. Los primeros datos del estudio MAGIC muestran que la combinación ECF (Epirrubicina, Cisplatino y 5 fluoruracilo en infusión continua) utilizada pre y postoperatoriamente aumenta la supervivencia libre de recidiva ${ }^{26}$. En la actualidad ya hay estudios en marcha que comparan las nuevas combinaciones de quimioterapia junto con radioterapia frente a regímenes similares al del estudio IT-0116.

\section{Bibliografía}

1. Ekstrom AM, et al. Decreasing incidence of both major histologic subtypes of gastric adenocarcinoma: A population-based study in Sweden. Br J Cancer 2000; 83:391-6.

2. Parkin DM, Pisani P, Ferlay J. Estimates of the worldwide incidence of 25 major cancers in 1990. Int J Cancer 1999; 80:827-41

3. Globocan URL disponible en: http://www-dep.iarc.fr/dataava/infodata.htm

4. Coleman MP, Gatta G, Verdecchia A, Esteve J, Sant M, Storm $\mathrm{H}$, Allemani C, Ciccolallo L, Santaquilani M, Berrino F, The EUROCARE Working Group. EUROCARE-3 summary: cancer survival in Europe at the end of the 20th century.Ann Oncol 2003; 14 Suppl 5:V128-V149.

5. Hundahl SA, Phillips JL, Menck HR. The National Cancer Data Base report on poor survival of U.S. gastric carcinoma patients treated with gastrectomy: fifth edition American Joint Committee on Cancer staging, proximal disease, and the "different disease" hypothesis. Cancer 2000; 88:921-32.

6. Hermans J, Bonenkamp JJ, Boon MC, et al. Adjuvant therapy after curative resection for gastric cancer: Meta-analysis of randomized trials. J Clin Oncol 1993; 11:1441-7.

7. Earle CC, Maroun JA. Adjuvant chemotherapy after curative resection for gastric cancer in non-Asian patients: Revisiting a meta-analysis of randomized trials. Eur J Cancer 1999; 35:1059-64.

8. Mari E, Floriani I, Tinazzi A, et al. Efficacy of adjuvant chemotherapy after curative resection for gastric cancer: A metaanalysis of published randomized trials. Ann Oncol 2000; 11:837-43.

9. Panzini I, et al. Adjuvant chemotherapy in gastric cancer: A meta-analysis of randomized trials and a comparison with previous meta-analyses. Tumori 2002; 88:21-7. 


\section{E. Vélez de Mendizábal y cols.}

10. Landry J, Tepper JE, Wood WC, Moulton EO, Koerner F, Sullinger J. Patterns of failure following curative resection of gastric cancer. Int J Radiat Oncol Biol Phys 1990; 191: 1357-62.

11. Macdonald JS, Smalley SR, Benedetti J, et al. Chemoradiotherapy after surgery compared with surgery alone for adenocarcinoma of the stomach or gastroesophageal junction. N Engl J Med 2001; 345:725-30.

12. Stomach. In: American Joint Committee on Cancer: AJCC Cancer Staging Manual. 6th ed. New York, NY: Springer 2002; 99-106.

13. Kajitani T, Japanese Research Society for the Study of Gastric Cancer. The general rules for gastric cancer study in Surgery and Pathology. Jpn J Surg 1981; 11:127-45.

14. Maruyama K, Sasako M, Kinoshita T, et al. Should systematic lymph node dissection be recommended for gastric cancer? Eur J Cancer 1998; 34:1480-90.

15. Nashimoto A, Nakajima $T$, Furukawa $\mathrm{H}$, et al: Randomized Trial of Adjuvant Chemotherapy With Mitomycin, Fluorouracil and Cytosine Arabinoside Followed by Oral Fluorouracil in Serosa Negative Gastric Cancer: Japan Clinical Oncology Group 9206-1. J Clin Oncol 2003; 21:2282-7.

16. Cuschieri A, Weeden S, Fielding J, et al. Patient survival after D1 and D2 resections for gastric cancer: Long-term results of the MRC randomized surgical trial-Surgical Co-Operative Group. Br J Cancer 1999; 79:1522-30.

17. Bonenkamp JJ, Hermans J, Sasako M, et al. Extended lymphnode dissection for gastric cancer: Dutch Gastric Cancer Group. N Engl J Med 1999; 340:908-14.

18. Bonenkamp JJ, Songun I, Hermans J, et al. Randomized comparison of morbidity after D1 and D2 dissection for gastric cancer in 996 Dutch patients. Lancet 1995; 345: 745-8.

19. Cornelis J.H. van de Velde, Koen C.M.J. Peeters. The Gastric Cancer Treatment Controversy. Journal of Clinical Oncology 2003; 21:2234-6.
20. Deguili $M$, Sasako $M$, Garino $M$, et al. Outcome of DI and D2 gastrectomy for gastric cancer: Preliminary results of the Italian Gastric Cancer Study Group (IGCSG) randomized controlled surgical trial. Presented at the XXXIII World Congress Int Coll Surg, Taipei, Taiwan, October 27-November 1, 2002

21. Schwarz RE, Zagala-Nevarez K. Recurrence Patterns After Radical Gastrectomy for Gastric Cancer: Prognostic Factors and Implications for Postoperative Adjuvant Therapy. Ann Surg Oncol 2002; 9:394-400.

22. Nitti $D$, Marchet A, Olivieri M, Ambrosi A, Mencarelli R, BeIluco C, Lise M. Ratio Between Metastatic and Examined Lymph Nodes Is an Independent Prognostic Factor After D2 Resection for Gastric Cancer: Analysis of a Large European Monoinstitutional Experience. Ann Surg Oncol 2003; 10: 1077-85.

23. Kapiteijn E, Marijnen CAM, Nagtegaal ID, Putter $H$, Steup WH, Wiggers T, Rutten HJ., Pahlman L, Glimelius B, van Krieken J HJM, Leer JWH, van de Velde CJH, The Dutch Colorectal Cancer Group Preoperative Radiotherapy Combined with Total Mesorectal Excision for Resectable Rectal Cancer. N Engl J Med 2001; 345:638-46.

24. Webb A, Cunningham D, Scarffe JH, et al. Randomized trial comparing epirubicin, cisplatin, and fluorouracil versus fluorouracil, doxorubicin, and methotrexate in advanced esophagogastric cancer. J Clin Oncol 1997; 15:261-7.

25. Kettner E, Ridwelski K, Keilholz U, et al. Docetaxel and cisplatin combination chemotherapy for advanced gastric cancer: Results of two phase II studies. Proc Am Soc Clin Oncol 2001; 20:165A (abstr).

26. Allum W, Cunningham D, Weeden S. Perioperative chemotherapy in operable gastric and lower esophageal cancer: A randomized, controlled trial (the MAGIC trial, ISRCTN 93793971). Proc Am Soc Clin Oncol 2003; 22: 249A (abstr).
Correspondencia:

Dra. E. Vélez de Mendizábal Sección de Oncología Médica Hospital San Millán San Pedro Avda. Autonomía de la Rioja, 3 E-26004 Logroño (La Rioja) 